\title{
Thrombectomy 6-24 hours after stroke in trial ineligible patients
}

\author{
Shashvat M Desai, ${ }^{1}$ Marcelo Rocha, ${ }^{1}$ Bradley J Molyneaux, ${ }_{1}^{1}$ Matthew Starr, ${ }^{1}$ \\ Cynthia L Kenmuir, ${ }^{1}$ Bradley A Gross, ${ }^{2}$ Brian Thomas Jankowitz, ${ }^{2}$ Tudor G Jovin, ${ }^{1,2}$ \\ Ashutosh P Jadhav ${ }^{1,2}$
}

${ }^{1}$ Department of Neurology, University of Pittsburgh Medical Center, Pittsburgh, Pennsylvania, USA

${ }^{2}$ Department of Neurosurgery, University of Pittsburgh, Pittsburgh, Pennsylvania, USA

\section{Correspondence to} Dr Ashutosh P Jadhav, Stroke Institute, University of Pittsburgh, Pittsburgh, PA 15218, USA; jadhavap@upmc. edu

Received 7 March 2018 Revised 2 May 2018 Accepted 2 May 2018 Published Online First 17 May 2018

Check for updates

To cite: Desai SM, Rocha M, Molyneaux BJ, et al. J Neurolntervent Surg 2018;10:1033-1037.

\section{ABSTRACT}

Background and purpose The DAWN and DEFUSE-3

trials demonstrated the benefit of endovascular thrombectomy (ET) in late-presenting acute ischemic strokes due to anterior circulation large vessel occlusion (ACLVO). Strict criteria were employed for patient selection. We sought to evaluate the characteristics and outcomes of patients treated outside these trials. Methods A retrospective review of acute ischemic stroke admissions to a single comprehensive stroke center was performed during the DAWN trial enrollment period (November 2014 to February 2017) to identify all patients presenting in the $6-24$ hour time window. These patients were further investigated for trial eligibility, baseline characteristics, treatment, and outcomes. Results Approximately $70 \%(n=142)$ of the 204 patients presenting 6-24 hours after last known well with NIH Stroke Scale score $\geq 6$ and harboring an ACLVO are DAWN and/or DEFUSE-3 ineligible, most commonly due to large infarct burden (38\%). $26 \%(n=37)$ of trial ineligible patients with large vessel occlusion strokes received off-label ET and 30\% of them achieved functional independence (modified Rankin Scale 0-2) at 90 days. Rates of symptomatic intracranial hemorrhage and mortality were $8 \%$ and $24 \%$, respectively

Conclusion Trial ineligible patients with large vessel occlusion strokes receiving off-label ET achieved outcomes comparable to DAWN and DEFUSE-3 eligible patients. Patients aged $<80$ years are most likely to benefit from ET in this subgroup. These data indicate a larger population of patients who can potentially benefit from ET in the expanded time window if more permissive criteria are applied.

\section{INTRODUCTION}

Acute ischemic stroke (AIS) due to large vessel occlusion (LVO) carries a poor prognosis in the absence of reperfusion therapy. Endovascular thrombectomy (ET) is the standard of care for acute ischemic stroke due to LVO. ${ }^{1}{ }^{2}$ Multiple randomized controlled trials reported in 2015 established superiority of ET over best medical management up to 12 hours from symptom onset. ${ }^{3-8}$ The DAWN trial (diffusion-weighted imaging or CT perfusion assessment with clinical mismatch in the triage of wake up and late presenting strokes undergoing neurointervention with Trevo) was strongly positive in favor of ET in the 6-24 hour time window, ${ }^{9}$ and the DEFUSE-3 trial (Endovascular Therapy Following Imaging Evaluation for Ischemic Stroke
3) was strongly positive in favor of ET in the 6-16 hour time window. ${ }^{10}$ Subsequently, American Heart Association 2018 guidelines offer level $1 \mathrm{~A}$ evidence for thrombectomy in the 6-16hour time window for patients meeting DAWN and/or DEFUSE-3 trial criteria and level 2A evidence for patients in the 16-24 hour window meeting DAWN criteria. $^{11}$

The DAWN and DEFUSE-3 trials had a significant treatment effect with a number needed to treat of 2.8 and 3.6, respectively. Adherence to selective trial-defined criteria in late time windows may deny ET to a population of patients who may benefit and prevent severe disability. Data regarding prevalence, treatments, and outcomes of LVOs not meeting DAWN and/or DEFUSE-3 criteria are limited. At our center we offer ET to patients who do not meet all trial criteria, after careful consideration by the vascular neurologist, neurointerventionalist, and consent from patients and their families. In this study we perform a single comprehensive stroke center analysis to clarify reasons for trial exclusion and safety and efficacy outcomes in patients not meeting trial criteria but receiving off-label ET.

\section{METHODS}

After institutional review board approval, a retrospective review of acute ischemic stroke admissions to a single comprehensive stroke center was performed during the DAWN enrollment period (November 2014 to February 2017) using the Get With The Guidelines database. Demographic characteristics, clinical and radiological data, treatment, and procedural information were extracted and analyzed.

\section{Patient selection}

Patients presenting 6-24hours after last known well (LKW) with a NIH Stroke Scale (NIHSS) score $\geq 6$ were identified. Further selection based on occlusion of a proximal intracranial internal carotid artery (ICA), middle cerebral artery segment 1 (MCA M1) or distal middle cerebral artery segment 2 (MCA M2) with or without extracranial occlusion was confirmed by CT angiography (CTA) and/ or magnetic resonance angiography (MRA) reports.

Patients were then screened for trial eligibility using DAWN/DEFUSE-3 trial criteria: NIHSS score $\geq 10($ DAWN) or NIHSS $\geq 6$ (DEFUSE-3); presence of proximal anterior circulation LVO; ischemic changes involving less than one-third of 
the MCA territory; presence of clinical-core mismatch (DAWN) or presence of target mismatch on perfusion imaging (DEFUSE3); baseline modified Rankin Scale (mRS) 0-1 (DAWN) or 0-2 (DEFUSE-3). ${ }^{9}$ Patients meeting the above criteria were then divided as follows: DAWN eligible, DEFUSE-3 eligible, and non-DAWN non-DEFUSE-3 (NDND) groups. Patients in the NDND group were categorized as NDND ET group and NDND non-ET group. Given the paucity of data regarding treatment of late presenting strokes, no prespecified treatment protocols were in place at our center. The decision to offer ET outside the DAWN/DEFUSE-3 trial was left to the discretion of the vascular neurologist and treating neurointerventionalist after detailed discussion with the patient and their families. Clinical guiding principles included: (1) ischemic changes involving less than one-third of the MCA territory; (2) NIHSS score $\geq 6$; (3) presence of proximal occlusion (ICA, M1, M2); (4) presence of significant mismatch (similar to a DAWN paradigm but including larger ischemic core volumes) between stroke severity and infarct burden (absence of a significant mismatch with little to no salvageable penumbra, as evident on perfusion imaging, is denoted as 'completed infarct'); (5) expected life expectancy $\geq 6$ months. Reasons for trial exclusion in the NDND group and reasons for clinical decision against ET in the NDND non-ET group were explored.

\section{Baseline characteristics}

Baseline demographic (age, sex), clinical (stroke severity, LKW, risk factor profile), radiographic (infarct volume, occlusion location) information and procedural technique and efficiency were compared between DAWN eligible, DEFUSE-3 eligible, and NDND ET groups. Infarct volume was quantified on CTP or MRI diffusion-weighted imaging using RAPID software (iSchemaView, Menlo Park, California, USA).

\section{Outcomes}

The primary safety end point was symptomatic intracranial hemorrhage $(\mathrm{sICH})$, defined as the presence of parenchymal hematoma type 2 on brain CT and/or MRI and neurological deterioration defined as an increase in NIHSS score of $\geq 4$ points within 36 hours from treatment. The secondary safety end point was stroke-related mortality. The efficacy end point was 90-day functional independence, defined as $\mathrm{mRS}$ score $0-2$. For patients who undergo thrombectomy, 90-day mRS scores are routinely ascertained via telephonic interview or during follow-up visit, and for patients who do not undergo thrombectomy, mRS data are obtained by review of follow-up, physical rehabilitation and/or mortality documentation conducted by trained research personnel not involved with patient treatment. The technical efficacy end point for patients who underwent ET was the rate of successful reperfusion, defined as Thrombolysis in Cerebral Infarction (TICI) score $\geq 2 \mathrm{~b}$.

\section{Statistical analyses}

Continuous variables are reported as mean \pm SD or median with interquartile range (as appropriate) and categorical variables are reported as proportions. Between-group comparison for continuous variables was performed using the Student's t-test and for categorical variables using the $\chi^{2}$ test or Fisher exact test, as appropriate. Univariable analysis and multivariable logistic regression analysis were performed to identify predictors and adjust for known confounders. Associations are presented as ORs with 95\%CI. Significance was defined as $\mathrm{P} \leq 0.05$.

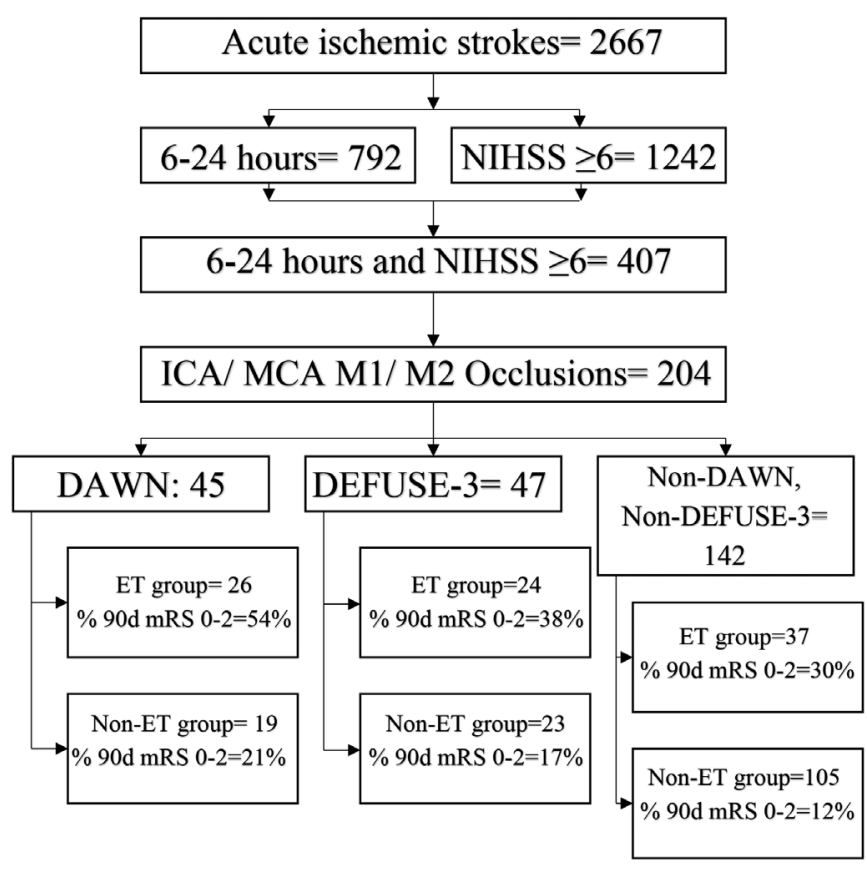

Figure 1 Flow chart for patient selection (note the overlap of 30 patients between the DAWN and DEFUSE-3 eligible groups).

Statistical analysis was performed using IBM SPSS Statistics 23 (IBM, Armonk, New York, USA).

\section{RESULTS}

\section{Patient selection}

A total of 2667 patients were identified with a discharge diagnosis of AIS. Of these, $30 \%$ of patients $(n=792)$ presented within the 6-24hour time window and 47\% $(n=1242)$ presented with NIHSS score $\geq 6 ; 15 \%$ of patients $(n=407)$ met both these criteria. Nearly half of these patients $(n=204)$ harbored a proximal anterior circulation LVO (ICA/M1/ M2; figure 1). Forty-five patients (22\%) met all DAWN criteria (DAWN eligible ET group, 26; non-ET group, 19) and 47 patients (23\%) met all DEFUSE-3 criteria (DEFUSE-3 eligible ET group, 24; non-ET group, 23). It is important to note that our center was a participating center in the DAWN trial but not in the DEFUSE-3 trial. Eight of the 45 patients who met DAWN trial criteria did not consent, so $37(82 \%)$ could be enrolled and randomized for the DAWN trial. Also, there was an overlap between DAWN and DEFUSE-3 eligible patients $(n=30)$. Of the 204 patients $(6-24$ hours since LKW, NIHSS score $\geq 6$, ICA/M1/M2 occlusion), 15 met the DAWN criteria only, 30 met the DAWN and DEFUSE-3 criteria, and 17 met only the DEFUSE-3 criteria.

The most common reasons for not meeting trial criteria (NDND group, $\mathrm{n}=142)$ included: ischemic core $>70 \mathrm{~mL}(38 \%)$, baseline $\mathrm{mRS}>2(27 \%)$, absence of clinical core mismatch or target mismatch on perfusion imaging (23\%), and/or distal occlusions (MCA-M2) (22\%). Thirty-seven (26\%) trial ineligible patients with LVO received off-label ET (NDND ET group). Reasons for not receiving endovascular therapy (NDND non-ET group, $\mathrm{n}=105)$ included: large ischemic core $(>70 \mathrm{~mL})$ in 51 patients $(49 \%)$, baseline $m R S>2(n=29,28 \%)$, completed infarct $(n=28,27 \%)$, and/or patient did not consent $(n=4,4 \%)$ (table 1). 
Table 1 Reasons for exclusion

\begin{tabular}{|c|c|}
\hline Reasons for trial ineligibility $(n=142)$ & $\mathrm{N}(\%)^{*}$ \\
\hline Infarct core volume $>70 \mathrm{~mL}$ & $54(38 \%)$ \\
\hline Baseline mRS >2 & $38(27 \%)$ \\
\hline $\begin{array}{l}\text { Absence of clinical core mismatch (DAWN) or target mismatch } \\
\text { (DEFUSE-3) } \dagger\end{array}$ & $32(23 \%)$ \\
\hline Distal occlusion (MCA-M2) & $31(22 \%)$ \\
\hline Reasons for not receiving off-label ET $(n=105)$ & $\mathrm{N}(\%) \ddagger$ \\
\hline Large infarct core $>70 \mathrm{~mL}$ & $51(49 \%)$ \\
\hline Baseline $\mathrm{mRS}>2$ & $29(28 \%)$ \\
\hline Completed infarct§ & $28(27 \%)$ \\
\hline Patient did not consent or opted against ET & $4(4 \%)$ \\
\hline \multicolumn{2}{|c|}{$\begin{array}{l}\text { * } 10 \% \text { of patients had more than one reason for trial ineligibility. } \\
+D A W N \text { : age } \geq 80 \text { years, NIHSS } \geq 10 \text { and infarct volume } \leq 21 \mathrm{~mL} \text {; age }<80 \\
\text { years, NIHSS } \geq 10 \text { and infarct volume } \leq 31 \mathrm{~mL} \text { or NIHSS } \geq 20 \text { and infarct } \\
\text { volume } \leq 51 \mathrm{~mL} \text {. DEFUSE- } 3 \text { : ischemic core volume }<70 \mathrm{~mL} \text {, mismatch ratio } \geq 1.8 \text { and } \\
\text { mismatch volume } \geq 15 \mathrm{~mL} \text {. } \\
\neq 8 \% \text { of patients had more than one reason for not receiving ET. } \\
\S \text { The term 'completed Infarct' used here implies that, while the ischemic core } \\
\text { volume }<70 \mathrm{~mL} \text {, there was little to no salvageable penumbra on perfusion imaging. } \\
\text { ET, endovascular thrombectomy; mRS, modified Rankin Scale; NIHSS, NIH Stroke } \\
\text { Scale. }\end{array}$} \\
\hline
\end{tabular}

\section{Baseline characteristics}

Baseline characteristics were compared between NDND ET, DAWN eligible, and DEFUSE-3 eligible groups (table 2).
Median infarct core volume was low $(<10 \mathrm{~mL})$ across all groups. The proportion of patients with coronary artery disease, witnessed stroke onset, tandem and distal occlusions (MCA-M2) was significantly higher in the NDND ET group. Also, LKW to arrival times were relatively shorter in the NDND ET group.

\section{Outcomes}

Technical efficacy

Almost half of the patients in the NDND ET group underwent ET using manual aspiration alone compared with only $8 \%$ of DAWN eligible and 21\% of DEFUSE-3 eligible patients. Rates of successful recanalization were high and comparable across groups ( $\geq 88 \%$ ) (table 3 ). More than $80 \%$ of NDND-ET patients underwent the procedure under intravenous conscious sedation.

\section{NDND-ET vs DAWN eligible ET vs DEFUSE-3 eligible ET}

Rates of early neurological recovery were significantly lower in the NDND ET group (24\%) compared with DAWN (58\%, $\mathrm{P}<0.01)$ and DEFUSE-3 (46\%, $\mathrm{P}=0.08)$ eligible groups. Functional independence (mRS 0-2 at 90 days) was 30\% in the NDND ET group, $54 \%$ in the DAWN ET group $(\mathrm{P}=0.05)$, and $38 \%$ in the DEFUSE-3 ET group $(\mathrm{P}=0.52)$ (figure 2). Rates of sICH (8\%; DAWN eligible 4\%, $\mathrm{P}=0.49$; DEFUSE-3 eligible $4 \%, \mathrm{P}=0.54)$ and mortality (24\%; DAWN eligible $15 \%$, $\mathrm{P}=0.38$; DEFUSE-3 eligible $13 \%, \mathrm{P}=0.25$ ) are shown in table 3 .

Rates of functional independence in trial eligible patients (DAWN and/or DEFUSE-3) who received ET $(n=38)$ were 53\%

\begin{tabular}{|c|c|c|c|c|c|c|}
\hline & $\begin{array}{l}\text { NDND ET group } \\
(n=37)\end{array}$ & $\begin{array}{l}\text { DAWN group } \\
(n=45)\end{array}$ & $\begin{array}{l}\text { P value } \\
\text { (DAWN vs NDND) }\end{array}$ & $\begin{array}{l}\text { DEFUSE-3 group } \\
(n=47)\end{array}$ & $\begin{array}{l}\text { P value (NDND } \\
\text { vs DEFUSE-3) }\end{array}$ & $\begin{array}{l}\text { NDND non- ET } \\
\text { group }(n=105)\end{array}$ \\
\hline \multicolumn{7}{|l|}{ Demographics } \\
\hline Age (median, IQR) & $77(62-86)$ & $73.5(61-83)$ & 0.46 & $75(61-85)$ & 0.96 & $67(59.5-82)$ \\
\hline Male sex & $51 \%(19)$ & $47 \%(21)$ & 0.74 & $38 \%(18)$ & 0.35 & $46 \%(48)$ \\
\hline \multicolumn{7}{|l|}{ Past medical history } \\
\hline Hypertension & $78 \%(29)$ & $60 \%(27)$ & 0.24 & $51 \%(24)$ & 0.01 & $69 \%(72)$ \\
\hline Diabetes mellitus & $22 \%(8)$ & $16 \%(7)$ & 0.45 & $19 \%(9)$ & 0.77 & $26 \%(27)$ \\
\hline Atrial fibrillation & $43 \%(16)$ & $27 \%(12)$ & 0.47 & $36 \%(17)$ & 0.50 & $41 \%(43)$ \\
\hline Tobacco & $24 \%(9)$ & $9 \%(4)$ & 0.05 & $13 \%(6)$ & 0.16 & $22 \%(23)$ \\
\hline Past stroke & $19 \%(7)$ & $11 \%(5)$ & 0.3 & $11 \%(5)$ & 0.28 & $11 \%(11)$ \\
\hline $\mathrm{CAD} / \mathrm{CHF}$ & $38 \%(14)$ & $18 \%(8)$ & 0.06 & $13 \%(6)$ & 0.007 & $14 \%(15)$ \\
\hline \multicolumn{7}{|l|}{ Clinical characteristics } \\
\hline Admission NIHSS score & $18(13-21)$ & $16(13-20)$ & 0.45 & $16(10-19)$ & 0.04 & $18(13-22)$ \\
\hline$\%$ Baseline mRS >2 & $24 \%(9)$ & $0 \%(0)$ & 0.0004 & $0 \%(0)$ & 0.0003 & $28 \%(29)$ \\
\hline LKW to arrival (min) & $539(432-766)$ & 707 (459-889) & 0.19 & $632(427-800)$ & 0.74 & 730 (480-991.5) \\
\hline$\%$ of witnessed strokes & $30 \%(11)$ & $11 \%$ & 0.03 & $11 \%$ & 0.02 & $21 \%(22)$ \\
\hline$\%$ of transfer patients & $78 \%(29)$ & $71 \%(32)$ & 0.45 & $68 \%(32)$ & 0.29 & $78 \%(82)$ \\
\hline \multicolumn{7}{|l|}{ Imaging characteristics } \\
\hline Infarct core volume $(\mathrm{mL})$ & $7(0-48)$ & $7(3-16)$ & 0.72 & $7(0-13)$ & 0.50 & $83(33.25-161.75)$ \\
\hline \multicolumn{7}{|l|}{ Site of occlusion§ } \\
\hline Intracranial ICA & $24 \%(9)$ & $16 \%(7)$ & 0.01 & $19 \%(9)$ & 0.01 & $10 \%(11)$ \\
\hline MCA-M1 & $57 \%(21)$ & $84 \%$ (38) & & $81 \%(38)$ & & $67 \%(70)$ \\
\hline MCA-M2 & $19 \%(7)$ & $0 \%(0)$ & & $0 \%(0)$ & & $23 \%(24)$ \\
\hline Tandem lesions & $27 \%(10)$ & $5 \%(2)$ & 0.003 & $15 \%(7)$ & 0.16 & $17 \%(18)$ \\
\hline
\end{tabular}

CAD, coronary artery disease; CHF, congestive heart failure; ICA, internal carotid artery; LKW, last known well; MCA, middle cerebral artery; M1 or M2, segment 1 or 2; NDND, non-DAWN non-DEFUSE-3 group. 
Ischemic Stroke

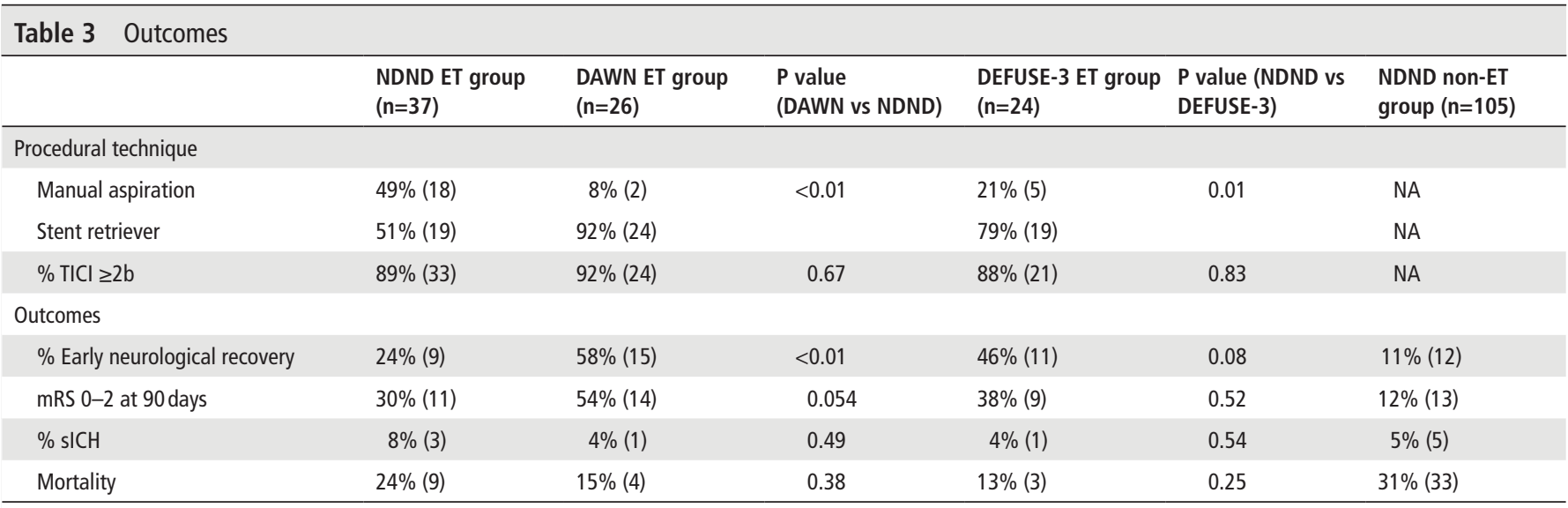

mRS, modified Rankin Scale; NDND, non-DAWN non-DEFUSE-3 group; sICH, symptomatic intracerebral hemorrhage; TICl, Thrombolysis in Cerebral Infarction.

$(n=20)$ compared with $30 \%(n=11)$ in the NDND ET group $(\mathrm{n}=37)(\mathrm{P}=0.04)$.

\section{NDND ET group}

Functional independence at 90 days after ET was observed in $30 \%$ of NDND ET group patients. This increased to $36 \%$ when patients with a baseline mRS of $0-2$ only were considered $(n=28)$. In patients $\geq 80$ years of age and pre-mRS $0-2$ $(n=8)$, rates of functional independence were very low $(13 \%$, $\mathrm{n}=1)$ with high mortality $(38 \%, \mathrm{n}=3)$. In patients with baseline ischemic core volume $<70 \mathrm{~mL}$ and baseline mRS $0-2(n=24)$, the rate of functional independence at 90 days was 38\% $(n=9)$.

\section{NDND non-ET group}

Functional independence at 90 days was achieved in $12 \%$ of patients $(n=13)$ who did not undergo ET compared with $30 \%$ $(n=11)$ in the NDND ET group $(\mathrm{P}=0.007)$. Rates of sICH were comparable (NDND non-ET 5\% vs NDND ET 8\%, P=0.44) but the mortality rate was slightly higher in the NDND non-ET group (31\% vs $24 \%, \mathrm{P}=0.41$ ).

\section{Predictors of good outcome}

In univariable analysis, age $(\mathrm{P}<0.001)$ and NIHSS score $(\mathrm{P}=0.01)$ were strong predictors of functional independence at
3 months in the NDND ET group. In a multivariable logistic regression model (age, sex, NIHSS score, occlusion location, LKW to arrival, infarct core volume, pre-mRS, mTICI grade), only successful reperfusion $(\mathrm{P}<0.001)$ was strongly associated with good outcomes.

\section{DISCUSSION}

Results of the DAWN (6-24 hours) and DEFUSE-3 (6-16 hours) trials have extended the therapeutic time limit for ET and shifted treatment eligibility to a physiology-based paradigm focusing on salvageable tissue. ${ }^{9} 10$ Both trials have demonstrated significant treatment effects and have proved that ET is superior to medical management in select patient populations in the late time window. Several factors contribute to such strong treatment effects: (1) higher than expected rates of functional independence in the treatment arm (DAWN 49\%, DEFUSE-3 45\%), in line with early window thrombectomy trials; and (2) very poor natural history of the control arm (rates of functional independence DAWN 13\%, DEFUSE-3 17\%) owing to absence of the systemic lytic agents in late time windows and very low rates of spontaneous recanalization, leading to continuous infarct growth as collateral blood supply eventually fails and is unable to support tissue viability.
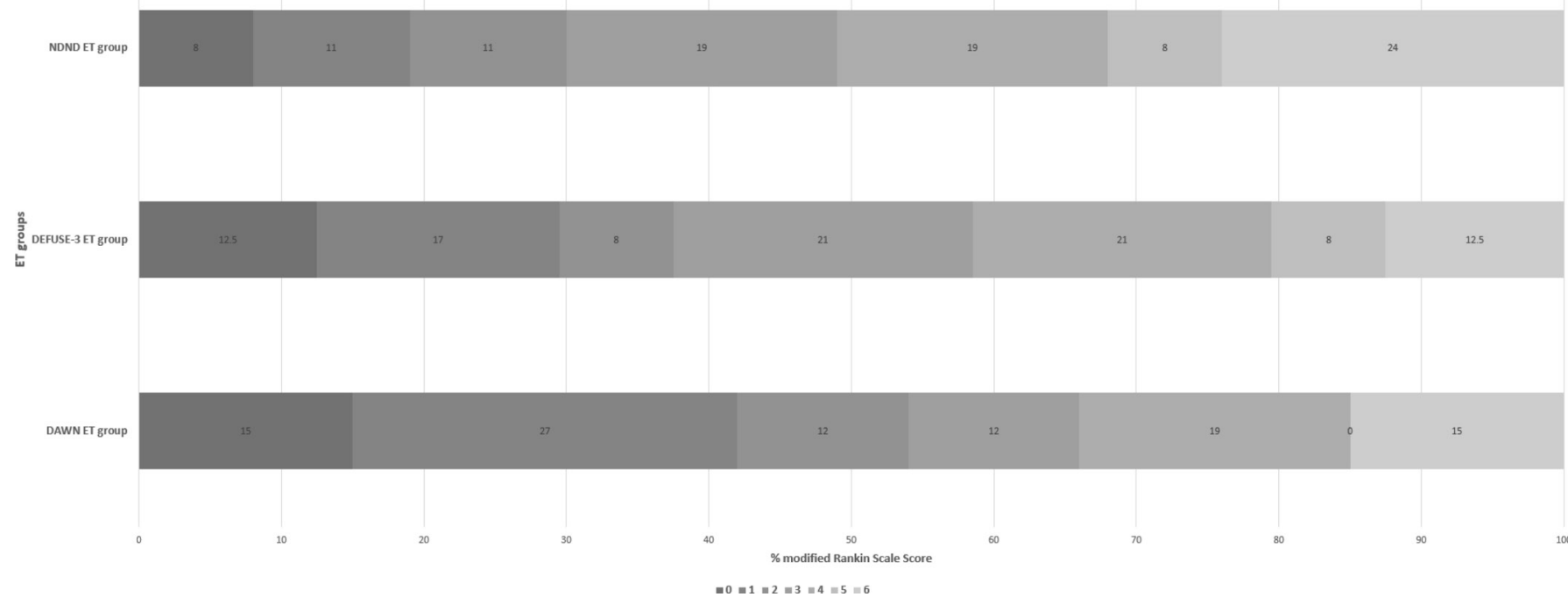

Figure 2 90-day modified Rankin Scale score across endovascular thrombectomy (ET) groups. NDND, non-DAWN non-DEFUSE-3 group. 
The inclusion criteria of the DAWN and DEFUSE-3 trials were very selective, limiting patient eligibility. In this study we found that approximately $70 \%$ of late-presenting LVO strokes did not meet either trial criteria (NDND group). The NDND group is composed of various patient populations. First, patients who would meet all DAWN criteria but were excluded due to NIHSS score 6-9, pre-mRS 2, and/or core more than $50 \mathrm{~mL}$ but less than $70 \mathrm{~mL}$. Second, patients who would meet all DEFUSE-3 criteria but were excluded due to presentation 16-24 hours since LKW and/or age $>90$ years. Third, patients with clinical core mismatch or target mismatch less than permissible per trial criteria and/ or distal occlusions (MCA-M2). Fourth, patients with core $>70 \mathrm{~mL}$ and/or pre-mRS $>2$. Trial exclusion occurred most commonly due to large infarct core $(>70 \mathrm{~mL})$, pre-mRS $>2$, absence of trial eligible salvageable tissue (penumbra), and/or MCA-M2 occlusion.

One-quarter of NDND patients received off-label ET and achieved functional independence in $30 \%$ of patients at 90 days with an acceptable safety profile; this number rises to $36 \%$ for patients with baseline mRS 0-2. Age and NIHSS are important predictors of good outcome and successful recanalization ( $\mathrm{mTICI} \geq 2 \mathrm{~b})$ is an independent predictor. Large infarct core $(>70 \mathrm{~mL})$, pre-mRS $>2$, and completed infarct with little or no penumbra were the most common reasons for NDND patients not receiving ET. Interestingly, rates of successful reperfusion in NDND-ET patients are comparable to DAWN ET patients despite using manual aspiration alone for half the patients.

The trials studied overlapping and mutually exclusive populations of patients; $33 \%$ of DAWN eligible patients do meet DEFUSE-3 criteria and 40\% of DEFUSE-3 patients do not meet DAWN criteria. ${ }^{12}$ In the DEFUSE-3 trial results, treatment effect is maintained in DAWN eligible and ineligible patients.

The DAWN trial allowed patients with an infarct core up to $51 \mathrm{~mL}$ and the DEFUSE-3 trial allowed patients with an infarct core up to $70 \mathrm{~mL}$. However, median infarct core volumes were low in DAWN $(\sim 9 \mathrm{~mL})$ and DEFUSE $3(\sim 10 \mathrm{~mL})$. The target cut-off infarct volume that predicts a good outcome decreases as age increases. ${ }^{13}$ Future studies are required to better define more permissive baseline ischemic core thresholds and further refine the full extent of clinical-core mismatch that leads to improved outcomes after ET.

Neither the DAWN nor the DEFUSE-3 trials included distal occlusions. A meta-analysis of 12 studies including 1080 patients with MCA-M2 occlusions has shown the benefit of ET in this subgroup in the early time window. ${ }^{14}$ A signal towards benefit is also evident in the HERMES meta-analysis. ${ }^{15}$ Mokin et al performed a multicenter retrospective study of M2 occlusions in which they found comparable outcomes in patients treated before and after 6 hours from symptom onset. ${ }^{16}$ Offering ET to this subgroup of patients, who otherwise have limited treatment options and a poor natural history, may be indicated. Also, in the future it will be important to study the safety and efficacy of ET in patients with NIHSS $<6$.

Relevant clinical end points may vary based on the patient's baseline functional status and natural history of the disease. For example, in patients with a baseline $\mathrm{mRS}>1$, a return to baseline functional status may be an acceptable outcome. Trials studying posterior circulation occlusions and $\mathrm{ET}^{17}$ and decompressive hemicraniectomy trials ${ }^{18}$ have used mRS 0-3 as a clinical end point. It is likely that a future ET trial for larger infarct core volumes will use a more liberal definition of acceptable clinical outcome. In our series we found a return to baseline rate of $53 \%$ in patients with baseline mRS 2 who underwent ET.
Only $13 \%$ of patients aged $\geq 80$ years in the NDND group with pre-mRS $0-2$ achieved functional independence and more than a third of them died before 90 days. This represents a very poor prognosis. These patients probably should therefore be selected for ET using more stringent criteria.

Our NDND patients who were not offered ET had a dismal natural history with only $12 \%$ achieving good outcome and a mortality rate of $31 \%$. This is likely due to the fact that this group had higher baseline ischemic core volume, higher baseline disability, and were not offered ET owing to a low likelihood of good outcome.

We recentlyreported that, of all acute ischemic strokes presenting to a comprehensive stroke center, $1.7 \%$ of patients met all DAWN criteria, 1.8\% met all DEFUSE-3 criteria, and $2.7 \%$ met DAWN and/or DEFUSE-3 criteria. This translates to about $9.2 \%$ of all ischemic strokes in the $6-24$ hour window meeting DAWN and/or DEFUSE-3 criteria, rising to $14 \%$ if the NDND-ET group is included. ${ }^{12}$

This study is limited by its retrospective nature, relatively small sample size, single center experience, and a clinical judgement-based selection process for treatment of patients not meeting trial criteria. Our analysis suggests that further liberalization of indications for ET in late time windows is achievable. Multicenter analysis and randomized controlled trials are necessary to validate our findings and define thresholds for ET to maximize indications and prevent long-term disability.

\section{CONCLUSION}

Strict adherence to DAWN and/DEFUSE-3 criteria would have denied ET to $18 \%(n=37)$ of the 204 patients who harbored an anterior circulation LVO and presented to our comprehensive stroke center within 6-24hours of LKW and with an NIHSS score $\geq 6$. Trial exclusion was most commonly attributed to large infarct core, poor baseline mRS, absence of clinical-core mismatch and/or distal occlusions. Our data indicate that ET may be safely offered to trial ineligible patients with less than trial-defined salvageable penumbra and/or MCA-M2 occlusions, particularly in patients with ischemic core volume of $<70 \mathrm{~mL}$, baseline mRS $0-2$, and age $\leq 80$ years. Natural history of untreated LVO patients in the late time window is poor and ET may be a favorable option, even for a subset of trial ineligible patients.

Contributors Conception and design: SMD, APJ. Acquisition of data: SMD. Analysis and interpretation of data: All: Drafting the article: SMD, APJ. Critically revising the article: All.:Administrative/technical/material support: All: Study supervision: APJ, TGJ.

Funding The authors have not declared a specific grant for this research from any funding agency in the public, commercial or not-for-profit sectors.

Competing interests TGJ: Consultant, Neuravi (steering committee - modest), Codman Neurovascular (DSMB - modest), Stryker Neurovascular (PI DAWN - unpaid), Fundacio Ictus (PI REVASCAT - unpaid). Stock: Anaconda, Silk Road, Blockade Medical (modest).

Patient consent Not required.

Ethics approval Institutional review board of University of Pittsburgh.

Provenance and peer review Not commissioned; externally peer reviewed.

(c) Article author(s) (or their employer(s) unless otherwise stated in the text of the article) 2018. All rights reserved. No commercial use is permitted unless otherwise expressly granted.

\section{REFERENCES}

1 Powers WJ, Derdeyn CP, Biller J, et al. 2015 American Heart Association/American Stroke Association focused update of the 2013 guidelines for the early management of patients with acute ischemic stroke regarding endovascular treatment: a guideline for healthcare professionals from the American Heart Association/American Stroke Association. Stroke 2015;46:3020-35. 
2 Wahlgren N, Moreira T, Michel P, et al. Mechanical thrombectomy in acute ischemic stroke: Consensus statement by ESO-Karolinska Stroke Update 2014/2015, supported by ESO, ESMINT, ESNR and EAN. Int J Stroke 2016;11:134-47.

3 Berkhemer OA, Fransen PS, Beumer D, et al. A randomized trial of intraarterial treatment for acute ischemic stroke. N Engl J Med 2015;372:11-20.

4 Jovin TG, Chamorro A, Cobo E, et al. Thrombectomy within 8 hours after symptom onset in ischemic stroke. N Engl J Med 2015;372:2296-306.

5 Goyal M, Demchuk AM, Menon BK, et al. Randomized assessment of rapid endovascular treatment of ischemic stroke. N Engl J Med 2015;372:1019-30.

6 Campbell BCV, Mitchell PJ, Kleinig TJ, et al. Endovascular therapy for ischemic stroke with perfusion-imaging selection. N Eng/ J Med Overseas Ed 2015;372:1009-18.

7 Saver JL, Goyal M, Bonafe A, et al. Stent-retriever thrombectomy after intravenous t-PA vs. t-PA alone in stroke. N Engl J Med 2015;372:2285-95.

8 Bracard S, Ducrocq X, Mas JL, et al. Mechanical thrombectomy after intravenous alteplase versus alteplase alone after stroke (THRACE): a randomised controlled trial. Lancet Neurol 2016;15:1138-47.

9 Nogueira RG, Jadhav AP, Haussen DC, et al. Thrombectomy 6 to 24 hours after stroke with a mismatch between deficit and infarct. N Engl J Med 2018;378:11-21.

10 Albers GW, Marks MP, Kemp S, et al. Thrombectomy for stroke at 6 to 16 hours with selection by perfusion imaging. N Engl J Med 2018;378:708-18.

11 Powers WJ, Rabinstein AA, Ackerson T, et al. 2018 guidelines for the early management of patients with acute ischemic stroke: a guideline for healthcare professionals from the American Heart Association/American Stroke Association. Stroke 2018;49:e46-e99.
12 Jadhav AP, Desai SM, Kenmuir CL, et al. Eligibility for endovascular trial enrollment in the 6- to 24-hour time window: analysis of a single comprehensive stroke center. Stroke 2018:49:1015-7.

13 Ribo M, Flores A, Mansilla E, et al. Age-adjusted infarct volume threshold for good outcome after endovascular treatment. J Neurointerv Surg 2014;6:418-22.

14 Saber H, Narayanan S, Palla M, et al. Mechanical thrombectomy for acute ischemic stroke with occlusion of the M2 segment of the middle cerebral artery: a metaanalysis. J Neurointerv Surg 2017. doi: 10.1136/neurintsurg-2017-013515. [Epub ahead of print].

15 Goyal M, Menon BK, van Zwam WH, et al. Endovascular thrombectomy after large-vessel ischaemic stroke: a meta-analysis of individual patient data from five randomised trials. Lancet 2016;387:1723-31.

16 Mokin M, Primiani CT, Ren Z, et al. Endovascular treatment of middle cerebral artery M2 occlusion strokes: clinical and procedural predictors of outcomes. Neurosurgery 2017;81:795-802.

17 Liu X, Xu G, Liu Y, et al. Acute basilar artery occlusion: endovascular interventions versus standard medical treatment (BEST) Trial-Design and protocol for a randomized, controlled, multicenter study. Int J Stroke 2017;12:779-85.

18 Vahedi K, Vicaut E, Mateo J, et al. Sequential-design, multicenter, randomized, controlled trial of early decompressive craniectomy in malignant middle cerebral artery infarction (DECIMAL Trial). Stroke 2007;38:2506-17. 